Maziuk Norbert, Szczypiór-Piasecka Karina, Mińko Alicja. Assessment of static and dynamic balance in patients after total hip arthroplasty based on the Tinetti Test. Journal of Education, Health and Sport. 2021;11(8):161-170. eISSN 2391-8306. DOI http://dx.doi.org/10.12775/JEHS.2021.11.08.016

https://apcz.umk.pl/czasopisma/index.php/JEHS/article/view/JEHS.2021.11.08.016

https://zenodo.org/record/5201568

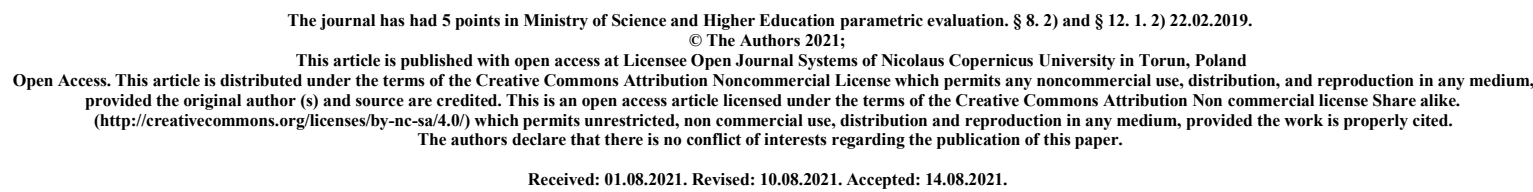

Assessment of static and dynamic balance in patients after total hip arthroplasty based on the Tinetti Test

\title{
Norbert Maziuk
}

Affiliation Department of Orthopaedic and Traumatology, Orthopaedic and

Traumatology Clinic Pomeranian Medical Medical University, ul. Unii

Lubelskiej 1, 71-252 Szczecin

Country Poland

Bio Statement

\section{Karina Szczypiór-Piasecka}

ORCID iD https://orcid.org/0000-0002-9562-9201

Affiliation Department of Orthopaedic and Traumatology, Orthopaedic and Traumatology Clinic Pomeranian Medical Medical University, ul. Unii

Lubelskiej 1, 71-252 Szczecin

Country

Bio Statement

\section{Alicja Mińko}

ORCID iD https://orcid.org/0000-0003-2299-3958 
Affiliation Department of Orthopaedic and Traumatology, Orthopaedic and Traumatology Clinic Pomeranian Medical Medical University, ul. Unii

\title{
Lubelskiej 1, 71-252 Szczecin
}

Country Poland

Bio Statement

Principal contact for editorial correspondence.

\begin{abstract}
Introduction: A degenerative disease of a hip joint (osteoarthritis) is a chronic affliction which causes progressive degeneration of a hip joint and tissue located around it. It inflicts limitation of locomotor skills, deteriorates life quality to a significant extent, and prevents a patient from normal activities in daily life. In Poland, more than 3 million people suffer from osteoarthritis. The disease symptoms are pain, limitation of mobility scope in a joint, postural and gait disorders, and positioning of a lower limb in non-functional position. The aim of the study was to analyze the gait pattern and to assess the static and dynamic balance of people qualified for hip joint arthroplasty, as well as possibility of falling.
\end{abstract}

Material and methods: The Tinetti Test has been used for the research in question. This test has been completed in patients awaiting for hip joint arthroplasty, and once more on a third day upon completion of arthroplasty, in the Department and Clinic of Orthopaedics, Traumatology and Oncology of Locomotor System of the Pomeranian Medical University in Szczecin. This test has included a group of $31-11$ women and 20 men.

Results: The mean age of the research group was 65.1 years. The intensity of pain in the hip joint, based on the VAS scale before and after the procedure, was 6.53 and 5.22 points, respectively. The average number of points obtained by the patient in the Tinetti Test before the surgery was 22.71 points. On the third day of hip surgery, the mean number of points decreased to 18.81 points.

Conclusions: Patients treated with alloplastic surgery of a hip joint show lower static and dynamic balance. Upon completion of the surgery, the patients are more prone to fall. The patients with completed hip joint arthroplasty show symptoms of locomotor system disorders. Key words: static balance; dynamic balance; total hip replacement; Tinetti Test 


\section{Introduction}

Osteoarthritis is the most common arthropathy that occurs in the human body. It causes multiple destructive changes and causes pain that impair human motor skills. In Poland, degenerative joint diseases occur in 8 million people, $40 \%$ of whom are related to the hip joint. Degenerative changes in joints are the result of mechanical and biological damage to the articular cartilage, the subcartilage layer of the bones and other structures that make up the joint. The lack of regenerative capacity of the hyaline cartilage is the cause of the irreversible and progressive nature of degenerative changes [1,2]. In the early stage of hip osteoarthritis, pain symptoms appear, initially aggravating after walking and transitory after rest. There is periodic limping and reduced mobility in the joint. As the disease progresses, there are night pains, pain when changing from sitting to standing position, functional shortening of the affected lower limb, disturbance of the gait pattern and reduced walking distance, and in the final stage, spontaneous stiffening of the hip joint may occur in a non-functional position of the lower limb $[3,4,5]$. The assessment of the clinical condition is possible thanks to imaging diagnostics, such as: radiological examination (X-ray), ultrasound examination (USG) or computed tomography (CT). The treatment of degenerative changes in the hip joint is divided into non-surgical and surgical treatment. The choice of the treatment method depends on the stage of the disease as well as the general and local condition of the patient [1]. In the early phase of the disease, the patient is qualified for non-surgical treatment based on pharmacotherapy and physiotherapy. In the case of ineffectiveness of non-operative treatment, surgical treatment is implemented. Three types of procedures are mainly used in osteoarthritis of the hip: osteotomy, arthrodesis and endoprosthesis [6,7].

Endoprosthetics is one of the most effective methods of treatment in terms of the patient's functioning and is the most frequently performed procedure in surgical treatment. It consists in removing the articular surfaces together with the bone elements that make up the joint and replacing them with an artificial joint. There are two types of arthroplasty: total and partial. Total arthroplasty consists in removing opposite articular surfaces and replacing them with implants. Total hip replacement consists of four elements: a stem, a acetabulum, a acetabular inlay and a head. Partial (hemi) arthroplasty involves replacing only the damaged articular surface. The hemispherical prosthesis consists of only with one component 
connected to the bone on one side and the other part used to recreate the shape of the articular surface $[1,8]$. Rehabilitation procedure should be carried out at every stage of the degenerative disease and should be adapted to the current condition of the patient. After arthroplasty, patients may experience disturbances in gait pattern and static and dynamic stabilization, therefore it is necessary to perform a thorough assessment to determine the degree of the above-mentioned dysfunctions $[9,10,11]$.

A useful research tool for the assessment of balance and gait is the Tinetti scale. It consists of two parts, and the result can be used to determine the tendency to fall. The performed assessment on the basis of the Tinetti scale allows the physiotherapist to implement appropriate therapy $[12,13]$.

The aim of the study was to assess the static and dynamic balance in patients qualified for hip arthroplasty. In addition, determining the likelihood of falls and the assessment of changes in gait in people after arthroplasty.

\section{Materials and methods}

The study involved 31 patients qualified for hip arthroplasty - 11 women and 20 men. The examination was performed on the day preceding the surgery and repeated on the third day after hip arthroplasty at the Department of Orthopedics, Traumatology and Oncology of the Musculoskeletal System of the Pomeranian Medical University in Szczecin. Until the surgery, the patients were not premedicated by anesthesia. The study was approved by the Bioethics Committee of the Pomeranian Medical University in Szczecin.

The standardized Tinetti Test was used to carry out the study. It is a scale that allows you to assess balance and gait. It consists of 16 tasks for which the patient receives from 0 to 2 points. In the first part, where static balance is assessed, the patient begins the test sitting on a hard chair without a handrail and performs 9 tasks (sitting balance, arises, attempts to arise, immediate standing balance, standing balance, nudged, eyes closed, turning $360^{\circ}$, sitting. down). The second part of the test includes 7 tasks (initiation of gait, step length and height, step symmetry, step continuity, path, trunk, walking stance), in which the patient's gait and dynamic balance are assessed with the use of standard orthopedic aids. The maximum number of points that can be obtained is 28-16 points from the first part and 12 points from the second part. Obtaining more than 24 points indicates a low risk of falls. A score of 19 to 24 indicates that the person is prone to falls. The respondent who scored below 19 points belongs to the group with a high probability of falls. The study also used the VAS scale, assessing the 
intensity of pain on a scale from 0 to 10 , where 0 means no pain, and 10 - the strongest pain. Using the VAS scale, pain in the hip joint was assessed before and after arthroplasty.

During the research, data was collected, which was archived using Microsoft Excel 2016 in Microsoft Office 2016 for Windows 10 and subjected to statistical analysis using STATISTICA 13 by StatSoft. Basic statistical measures were computed to analyze the variables in the study. The results are presented in the form of mean values, median and standard deviation. During the observation of the statistical relationships that occurred between inconsistent variables, the Pearson correlation coefficient was applied and the static significance was calculated for variables with a normal distribution and order - student's $\mathrm{T}$ test. with a normal distribution, the analysis was performed using the t-test for paired samples and the t-test for independent samples. The analysis of non-normal distributions was computed using nonparametric statistical tests. The statistical analyzes used in the study determined the significance coefficient " $p$ " with a value of $p \leq 0.05$.

\section{Results}

The mean age of the research group was 65.1 years. The average body weight of the patients is $81.1 \mathrm{~kg}$, and the average height is $170.7 \mathrm{~cm}$. The average BMI of the examined people was 27.7. The male and female metrics are shown in Table 1.

Table 1. Characteristics of the group

\begin{tabular}{|c|c|c|c|c|c|}
\hline \multicolumn{2}{|c|}{} & Age & Body weight & Growth & BMI \\
\hline \multirow{3}{*}{ Total } & $\bar{x}$ & 65,1 & 81,1 & 170,7 & 27,7 \\
\cline { 2 - 7 } & $\mathrm{Me}$ & 65 & 78 & 170 & 27,47 \\
\cline { 2 - 7 } & $\mathrm{SD}$ & 9,3 & 16,8 & 9,1 & 4,61 \\
\hline \multirow{4}{*}{ Women } & $\bar{x}$ & 67,2 & 70,5 & 161,6 & 27,12 \\
\cline { 2 - 7 } & $\mathrm{Me}$ & 67 & 67 & 164 & 24,8 \\
\cline { 2 - 7 } & $\mathrm{SD}$ & 8,2 & 12,3 & 4,8 & 5,38 \\
\hline \multirow{3}{*}{ Men } & $\bar{x}$ & 64 & 86,9 & 175,7 & 28,02 \\
\cline { 2 - 7 } & $\mathrm{Me}$ & 65 & 85,5 & 176 & 27,75 \\
\cline { 2 - 7 } & $\mathrm{SD}$ & 9,9 & 16,3 & 6,7 & 4,24 \\
\hline
\end{tabular}

Legend: $\bar{x}_{\text {- mean; }} \mathrm{Me}$ - median; SD - standard deviation; BMI - Body Mass Index

Table 2 presents the values of the intensity of pain in the hip joint, which were determined by the patients using the VAS scale before and after the procedure. The mean value before arthroplasty was 6.53 , and after arthroplasty -5.22 . 
Table 2. Assessment of pain using the VAS scale

\begin{tabular}{|l|c|c|c|c|}
\hline & $\bar{x}$ & Me & SD & p-value \\
\cline { 1 - 3 } Before the surgery & 6,53 & 8 & 2,14 & \multirow{2}{*}{0,989} \\
\cline { 1 - 3 } After the surgery & 5,22 & 5 & 2,36 & \\
\hline
\end{tabular}

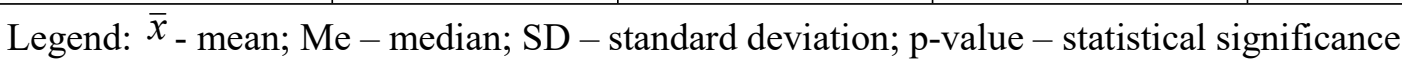

The static and dynamic balance of patients qualified for hip arthroplasty was analyzed using the Tinetti Test scale. In the first part of the study, which concerned the assessment of static equilibrium, the patients obtained an average of 13.5 points out of 16 possible points before the procedure. On the third day after the surgery, the mean score dropped to 11.6 points. In the second stage, where the dynamic balance was assessed, the subjects examined before arthroplasty achieved an average of 9.66 points out of 12 points. The average result after 3 days after the procedure was 7.6 points The average number of points scored by the patient before the surgery was 22.71 points On the third day of hip surgery, the mean number of points decreased to 18.81 points. Detailed results for individual trials are presented in Table 3.

Table 3. Tinetti Test results before and after arthroplasty

\begin{tabular}{|c|c|c|c|c|c|c|c|c|}
\hline & \multicolumn{3}{|c|}{ Before arthroplasty } & \multicolumn{3}{|c|}{ After arthroplasty } & \multirow{2}{*}{$\begin{array}{c}\text { p- } \\
\text { value }\end{array}$} \\
\hline & & $\bar{x}$ & Me & SD & $\bar{x}$ & Me & SD & \\
\hline \multirow{10}{*}{ Part I } & Sitting balance & 0,97 & 1 & 0 & 0,97 & 1 & 0 & 0.326 \\
\hline & Arises & 1,22 & 1 & 0,44 & 0,97 & 1 & 0,26 & 0,998 \\
\hline & Attempts to arise & 1,9 & 2 & 0,18 & 1,75 & 2 & 0,48 & 0,955 \\
\hline & Immediate standing balance & 1,66 & 2 & 0,46 & 1,09 & 1 & 0,43 & $>0,999$ \\
\hline & Standing balance & 1,63 & 2 & 0,48 & 1,16 & 1 & 0,48 & $>0,999$ \\
\hline & Nudged & 1,94 & 2 & 0 & 1,88 & 2 & 0,36 & $>0,999$ \\
\hline & Eyes closed & 0,97 & 1 & 0 & 0,94 & 1 & 0,18 & 0,747 \\
\hline & Turning $360^{\circ}$ & 0,94 & 1 & 0,18 & 0,94 & 1 & 0,18 & 0,326 \\
\hline & Sitting down & 1,31 & 1 & 0,49 & 1 & 1 & 0,18 & 0,999 \\
\hline & Total 1 & 13,5 & 14 & 1,55 & 11,59 & 12 & 1,54 & $>0,999$ \\
\hline \multirow{12}{*}{$\begin{array}{l}\text { Part } \\
\text { II }\end{array}$} & Initiation of gait & 0,97 & 1 & 0 & 0,97 & 1 & 0 & 0.326 \\
\hline & \multirow{2}{*}{$\begin{array}{l}\text { Step length and height - right } \\
\text { leg }\end{array}$} & 0,91 & 1 & 0,25 & 0,75 & 1 & 0,43 & 0,982 \\
\hline & & 0,97 & 1 & 0 & 0,97 & 1 & 0 & 0.326 \\
\hline & \multirow{2}{*}{$\begin{array}{l}\text { Step lenght and height - left } \\
\text { leg }\end{array}$} & 0,91 & 1 & 0,25 & 0,72 & 1 & 0,44 & 0,991 \\
\hline & & 0,97 & 1 & 0 & 0,94 & 1 & 0,18 & 0,747 \\
\hline & Step symmetry & 0,75 & 1 & 0,43 & 0,38 & 0 & 0,5 & $>0.999$ \\
\hline & Step continuity & 0,97 & 1 & 0 & 0,94 & 1 & 0,18 & 0,747 \\
\hline & Path & 1,54 & 2 & 0,5 & 0,97 & 1 & 0 & $>0,999$ \\
\hline & Trunk & 0,75 & 0 & 0,99 & 0 & 0 & 0 & $>0,999$ \\
\hline & Walking stance & 0,97 & 1 & 0 & 0,97 & 1 & 0 & 0,326 \\
\hline & Total 2 & 9,66 & 10 & 1,7 & 7,59 & 8 & 1,04 & $>0,999$ \\
\hline & Total 1 and 2 & 22,72 & 24 & 3,57 & 18,81 & 20 & 2,91 & $>0,999$ \\
\hline
\end{tabular}

Legend: - mean; $\mathrm{Me}$ - median; SD - standard deviation; $\mathrm{p}$-value - statistical significance 


\section{Discussion}

The Tinetti Test scale was used in the study. The aim of the study was to assess the parameters of static and dynamic balance in patients qualified for hip arthroplasty, to determine the risk of falls and to analyze the locomotor functions.

A similar study was carried out by Bennett D. and co-authors who assessed the ability to walk at a length of 10 meters in 95 patients on day 2 after hip replacement surgery. The researchers noticed that the stride length with the operated leg was reduced in relation to the non-operated leg, which disturbed the symmetry and the correct gait pattern [14]. Similar results can be observed in this study.

Majewski M. and co-authors conducted a study in 2005 assessing balance control in patients after hip arthroplasty while standing, walking and changing body position from sitting to standing using sensors on the body of the subjects. The research group consisted of 25 patients - 11 women and 14 men. They were examined before the procedure and 4 and 12 months after the procedure. For comparison, 50 healthy people were examined as a control group. Before the surgery, the control of balance while walking and changing body position was weaker than in the control group. The standing balance test showed no significant difference between the groups. After 4 and 12 months after arthroplasty, patients showed gradual improvement in maintaining balance and needed less time to perform tasks. Researchers noticed reduced trunk stability in patients after surgery compared to the control group during walking [15]. The present study also demonstrates weakened static balance and postoperative gait disturbance.

In 2003, Nallegowda M. and co-authors examined a group of 30 patients after hip arthroplasty in order to assess their balance and gait. The control group included 30 people of the same age and gender. Dynamic posturography was used to investigate the equivalent functions. In patients after arthroplasty, compared to the control group, no loss of proprioception was observed. It was found that, however, they required a greater proportion of the visual organ and the vestibular system to maintain balance and a delayed motor response. In the case of gait, they used a compensatory strategy. Researchers concluded that postoperative people require balance, gait and daily activities training. The results of this study also showed a disturbance in the gait pattern in postoperative patients and an incorrect body posture during locomotion [16].

The study by Pop T. and co-authors included a group of 55 patients in the period of 23 years after total hip arthroplasty, and a control group - 48 healthy subjects corresponding to 
the patient's age. The test consisted in maintaining a standing position of both feet on a stabilographic platform with eyes closed and open. The static equilibrium of the research group was significantly lowered compared to the control group, especially during the eyesclosed task. The present study showed an imbalance in the static balance when maintaining a standing posture and immediately after changing the body position from sitting to standing. Closing the eyes by patients did not significantly affect the results of the study [17].

In a study by Rita R. et al. from 2012, the gait parameters of patients after hip arthroplasty were assessed. The study included 80 patients after arthroplasty and a control group - 40 healthy people and was carried out 3,6 and 12 months after the procedure. A computer system of traffic analysis was used for the study. People from the control group achieved very good gait parameters. In patients after hip replacement surgery, a statistically significant improvement in the locomotor function was observed 12 months after the procedure. The present study reveals the deterioration of locomotor functions in postoperative patients, which was influenced by disturbances in gait symmetry and the length of steps taken, as well as the presence of torso deflections during movement. The reason for the differences in the analysis of gait parameters may be the early performance of the examination - 3 days after the surgery [18].

In 2008, Lugade V. and co-authors conducted research activities to evaluate the control of body balance during gait after hip arthroplasty using a computer system that analyzes movement along a 10-meter path. A total of 30 people, divided into two groups, participated in the study. The first group consisted of 20 patients - 14 men and 6 women, qualified for hip arthroplasty. The second group was a control group - 10 healthy people of similar age. The examination was performed before the operation and 4 months after the operation. Patients after arthroplasty significantly improved body balance after 4 months. The persistence of deficits during locomotion was found compared to the control group [19]. As a result of this study, we present the deterioration of locomotor functions in postoperative patients in the form of changes in the length of the steps taken and the failure to maintain gait symmetry. However, the study was conducted earlier after arthroplasty, which may be the reason for the differences in the results.

\section{Conclusion}

Patients undergoing hip arthroplasty show a reduction in the parameters of static and dynamic balance. Patients after hip arthroplasty have a higher risk of falls in the early postoperative period. Patients after hip arthroplasty suffer from abnormal gait function. 


\section{Literature:}

1. Nowakowski A, Mazurek T. Ortopedia i traumatologia. Poznań: Wydaw. Naukowe Exemplum; 2017. ISBN 978-83-62690-28-2.

2. Białoszewski D. Fizjoterapia w ortopedii. Warszawa: Wydaw. Lekarskie PZWL; 2015. ISBN 978-83-200-4802-5.

3. Błaszczyk JW. Biomechanika kliniczna. Warszawa: Wydaw. Lekarskie PZWL; 2004. ISBN 978-83-200-3748-7.

4. Kusz D. Kompendium ortopedii. Warszawa: Wydaw. Lekarskie PZWL; 2009. ISBN 97883-200-3765-4.

5. Coote A, Haslam P. Ortopedia i reumatologia. Wrocław: Wydaw. Elsevier Urban \& Partner; 2007. ISBN 978-83-89581-84-6.

6. Murphy NJ, Eyles JP, Hunter DJ. Hip Osteoarthritis: Etiopathogenesis and Implications for Management. Adv Ther. 2016;33(11):1921-1946. doi: 10.1007/s12325-016-0409-3.

7. Lespasio MJ, Sultan AA, Piuzzi NS, Khlopas A, Husni ME, Muschler GF, Mont MA. Hip Osteoarthritis: A Primer. Perm J. 2018;22:17-084. doi: 10.7812/TPP/17-084.

8. Elias-Jones C., Perry M.: Ortopedia i reumatologia. Wrocław: Wydaw. Edra Urban \& Partner; 2013. ISBN 978-83-65373-00-7.

9. Bennell K. Physiotherapy management of hip osteoarthritis. J Physiother. 2013;59(3):14557. doi: 10.1016/S1836-9553(13)70179-6.

10. Di Monaco M, Vallero F, Tappero R, Cavanna A. Rehabilitation after total hip arthroplasty: a systematic review of controlled trials on physical exercise programs. Eur J Phys Rehabil Med. 2009;45(3):303-17.

11. Skou ST, Roos EM. Physical therapy for patients with knee and hip osteoarthritis: supervised, active treatment is current best practice. Clin Exp Rheumatol. 2019;120(5):112117.

12. Curcio F, Basile C, Liguori I, Della-Morte D, Gargiulo G, Galizia G, Testa G, Langellotto A, Cacciatore F, Bonaduce D, Abete P. Tinetti mobility test is related to muscle mass and strength in non-institutionalized elderly people. Age (Dordr). 2016;38(5-6):525-533. doi: 10.1007/s11357-016-9935-9.

13. Kloos AD, Fritz NE, Kostyk SK, Young GS, Kegelmeyer DA. Clinimetric properties of the Tinetti Mobility Test, Four Square Step Test, Activities-specific Balance Confidence Scale, and spatiotemporal gait measures in individuals with Huntington's disease. Gait Posture. 2014;40(4):647-51. doi: 10.1016/j.gaitpost.2014.07.018. 
14. Bennett D, Ogonda L, Elliott D, Humphreys L, Lawlor M, Beverland D. Comparison of immediate postoperative walking ability in patients receiving minimally invasive and standard-incision hip arthroplasty: a prospective blinded study. J Arthroplasty. 2007;22(4):490-5. doi: 10.1016/j.arth.2006.02.173.

15. Majewski M, Bischoff-Ferrari HA, Grüneberg C, Dick W, Allum JH. Improvements in balance after total hip replacement. J Bone Joint Surg Br. 2005;87(10):1337-43. doi: 10.1302/0301-620X.87B10.16605.

16. Nallegowda M, Singh U, Bhan S, Wadhwa S, Handa G, Dwivedi SN. Balance and gait in total hip replacement: a pilot study. Am J Phys Med Rehabil. 2003;82(9):669-77. doi: 10.1097/01.PHM.0000083664.30871.C8.

17. Pop T, Szymczyk D, Majewska J, Bejer A, Baran J, Bielecki A, Rusek W. The Assessment of Static Balance in Patients after Total Hip Replacement in the Period of 2-3 Years after Surgery. Biomed Res Int. 2018;4;2018:3707254. doi: 10.1155/2018/3707254.

18. Kiss RM, Illyés Á. Comparison of gait parameters in patients following total hip arthroplasty with a direct-lateral or antero-lateral surgical approach. Hum Mov Sci. 2012;31(5):1302-16. doi: 10.1016/j.humov.2012.02.004.

19. Lugade V, Klausmeier V, Jewett B, Collis D, Chou LS. Short-term recovery of balance control after total hip arthroplasty. Clin Orthop Relat Res. 2008;466(12):3051-8. doi: 10.1007/s11999-008-0488-9. 